\title{
Measurements of neutral gas fluxes under different plasma and divertor regimes in ASDEX Upgrade
}

\author{
A. Scarabosio*, G. Haas, H.W. Müller, R. Pugno, \\ M. Wischmeier and the ASDEX Upgrade Team \\ Max-Planck-Institut für Plasmaphysik, EURATOM-Association, Boltzmannstr. 2, \\ D-85748, Garching, Germany
}

\begin{abstract}
We report on neutral flux measurements in the full tungsten ASDEX Upgrade from the recently upgraded pressure gauge system in a wide range of plasma parameters and divertor conditions in L-mode plasmas. We compare these measurements with the ion and neutral fluxes in similar older discharges in presence of carbon target plates. We then show the qualitative relationship between the divertor poloidal distribution of the neutral flux density and the inner and outer divertor plasma regimes. During plasma density rise, when the plasma at one particular target detaches, the local flux increases abruptly producing a strong in-out asymmetry in the neutral flux distribution in the divertor. The neutral flux at the high field side increase linearly with the plasma density whereas at the low field side mid-plane it remains negligible in all the cases considered here. Finally, we compare detailed ion and neutral flux density profiles along the outer target from Langmuir probes, $D_{\alpha}$ radiation and particle flux measurements obtained during vertical strike point sweeps.
\end{abstract}


Key words: ASDEX-Upgrade, Edge plasma, Divertor, Divertor neutrals PACS: 52.25.Ya, 52.55.Fa, 52.25.-b

\section{Introduction}

Up to date, the integrated modelling of the scrape-off-layer (SOL), divertor plasma and neutral gas does not fully reproduce the experimental observations in present day machines [1], thus leading to large uncertainties in the predictions for future devices such as ITER. One key element, essential for the understanding of divertor physics and the improvement of the modeling, is the role of the neutral gas. This paper reports on detailed neutral flux measurements in the divertor of the ASDEX Upgrade tokamak in order to document conditions and to establish a database for future edge code validation $[1,2]$.

The fast neutral gas diagnostic comprises 20 ionisation gauges of the ASDEXtype [3], located in different toroidal and poloidal positions covering the upper and lower divertor and main chamber (see figure 1) and has a time resolution of $1 \mathrm{~ms}$. The gauge head, installed in a box with a small entrance hole, measures directly the neutral particle density, $n_{0}$, in the volume between the acceleration grid and the ion collector (ionisation volume). This density is, in general, different from the external density. Through proper calibration however, the gauge signal can be related to the neutral flux density outside the gauge, $\Gamma_{D}$, without any assumption on the neutrals velocity distribution if the conduc-

* Max-Planck-Institut für Plasmaphysik, EURATOM-Association, Boltzmannstr. 2, D-85748, Garching, Germany

Email address: andrea.scarabosio@ipp.mpg.de (the ASDEX Upgrade Team). 
tance into the ionisation volume is sufficiently small [3]. Recently, in 2006 and 2007, new gauges have been installed. In particular, gauges \#20 and \#8 are connected to the front of the target plate through a chimney in between tiles in the inner and outer divertor respectively and measures directly the recycling in front of the targets. Due to the size of the chimney and the geometry of tiles the measurement is localised in $1-1.5 \mathrm{~cm}$ around the chimney position. The spectroscopic measurements of $D_{\alpha}$ radiation in the divertor region uses many lines of sight connected via fiber optics to a spectrometer. The system has a typical time resolution of $100 \mu \mathrm{s}$ and about $2 \mathrm{~cm}$ of spatial resolution. Several Langmuir probes, flush mounted in the divertor tiles, provide electron density/temperature and the ion fluxes, $\Gamma_{+}$, at the targets. A set of ohmic and ECRH heated L-mode discharges at different line averaged densities, $\bar{n}_{e}$, were used to obtain the poloidal distributions of neutral fluxes. We mainly concentrate on the divertor region. In fact the neutral fluxes at the outer mid-plane is, in these discharges, always below $4 \times 10^{20}$ particle/ $s$, practically negligible with respect to divertor values. Density variation and detachment are achieved through gas puffing from the low-field-side main chamber or from the lower divertor, below the roof baffle. The gas is puffed, respectively, from one (sector 1 of 16) and 8 toroidally equidistant locations (sectors 2, 4, 6, 8, 10, 12, 14 , and 16). The influx rate, $\Gamma_{\text {influx }}=0.5-20 \times 10^{21}$ particle $/ \mathrm{s}$, is typically more than 20 times smaller than the fluxes from recycling in the sub-divertor. In both cases no toroidal asymmetry in the neutral gas fluxes are observed in the 5 toroidally distributed "subdivertor" gauges (figure 1) installed below the dome (sectors 3,7 and 15). This is likely explained by the fact that neutral fluxes in the divertor are dominated by recycling. At present, all plasma facing components (PFCs) in ASDEX Upgrade are made of W-coated carbon tiles [4]. The new results from 2007 presented here are compared with the ones 
from 2006 campaign obtained with pure carbon targets. During the flattop phase of the discharges, an outer strike point sweep allowed the measurement of the neutral flux profile at the outer target near the separatrix. The neutral flux profiles so obtained are compared with the ion flux from the Langmuir probes and with the neutral particle fluxes obtained from $D_{\alpha}$ measurements. Under the assumption of dominant surface recombination the $D_{\alpha}$ radiation is emitted locally in front of the target. The $T_{e}$ and $n_{e}$ from the Langmuir probes can then be used to calculate the photon efficiency coefficient S/XB and thus the neutral fluxes. This approach is expected to be valid in attached plasma condition with $T_{e}$ at target larger than $15 \mathrm{eV}$ with the $D_{\alpha}$ emission originating only from a layer in front of the target plate and not along the divertor leg.

\section{Poloidal distribution of the neutral gas flux density and the con- nected recycling regions in the inner and outer divertor.}

The peak ion flux to the outer target, the correspondent $T_{e}$ and the neutral flux in the subdivertor in a set of $1 M A$ discharges are shown as functions of the line averaged density $\bar{n}_{e}$ in figure 2 . The dots represent plasmas with $\mathrm{W}$ targets whereas the diamonds have CFC targets. The two sets of data look very similar. For the same $\bar{n}_{e}$ the two sets of discharges have almost identical upstream parameters. With $\mathrm{W}$ the drop in the ion flux, characteristic of detachment together with a low electron temperature $T_{e}<5 \mathrm{eV}$ [5], appears for $\bar{n}_{e}$ between 5.5 and $6 \times 10^{19} \mathrm{~m}^{-3}$. With CFC the threshold density for detachment appears to be similar within $10 \%$. In general, this result reflects the small difference observed in the neutral gas dynamic between $\mathrm{W}$ and $\mathrm{CFC}$ target plates. We note that at the highest density, the neutral fluxes for the 
CFC targets is higher by a factor of 2 . This difference in recycling level cannot be attributed directly to the different PFC materials since some experimental conditions were also different. For instance we used main chamber fueling for the full-W series and lower divertor fueling for CFC plates. In this latter case the pumping capability was also lower by about $30 \%$ compared to the actual value of $155 \mathrm{~m}^{3} \mathrm{~s}^{-1}$.

It is well know that the neutral flux in the divertor strongly increases with $\bar{n}_{e}[6,7]$ as in figure 2. However, we show that $\Gamma_{D}$ is not a smooth function of $\bar{n}_{e}$. Sudden and rapid changes appear for small variations of the plasma density, and are generally correlated with transitions in the divertor plasma regime as we describe by the following examples. A discharge with $1 M A$, $\bar{n}_{e}=1 \times 19 m^{-3}$ and $0.7 M W \mathrm{ECRH}$ is shown in figure 3 . As in all the discharges presented here the $\mathrm{X}$-point was formed at $\mathrm{t}=0.5 \mathrm{~s}$. During the low density phase, the plasma at the inner target is relatively hot, $T_{e}>20 \mathrm{eV}$, indicating an attached state. The outer divertor shows higher $T_{e} \sim 35 \mathrm{eV}$ and it is also attached. The neutral flux density is greatest in the subdivertor region, it is about twice the inner divertor flux and four times the outer divertor flux, $\Gamma_{D, F 1} \sim 2 \Gamma_{D, F 19} \sim 4 \Gamma_{D, F 8}$. At this relatively high $T_{e}$ the plasma in front of the targets is opaque to neutrals from the private flux region and the gauges only see the local recycling fluxes. Subsequently, the $\bar{n}_{e}$ is ramped up. The $\bar{n}_{e}$ rise leads to a fast increase of the neutral fluxes at the inner target and subdivertor which are now comparable $\Gamma_{D, F 1} \approx \Gamma_{D, F 19}$. Langmuir probe data show $T_{e}<5 \mathrm{eV}$ and $n_{e} \sim 1 \times 10^{19} \mathrm{~m}^{-3}$ at the inner target indicating detached plasma whilst at the outer target stays attached with $T_{e} \sim 25 \mathrm{eV}$. The flux equalisation $\Gamma_{D, F 1} \approx \Gamma_{D, F 19}$ may be understood considering the low $T_{e}$ in the detached inner target plasma which is now more transparent to neutral 
particles. The neutrals can cross the inner divertor leg without being ionised. This condition would likely lead to the stronger poloidal asymmetric neutral distribution observed in the experiment with $\Gamma_{D, F 19} \approx 3 \Gamma_{D, F 8}$. Fluxes at outer target and HFS main chamber increase more moderately, almost linearly with $\bar{n}_{e}$, indicating that the majority of the recycling is now concentrated along the inner target. Under this condition, the large recycling at the inner target dominates the neutrals contribution to the subdivertor.

At higher density $\left(\bar{n}_{e}>5.5 \times 10^{19} \mathrm{~m}^{-3}\right)$ the situation changes further. Figure 4 shows a 1 MA ohmic discharge. Due to the lower input power the inner target is already fully detached during the density ramp with $T_{e} \sim 5 \mathrm{eV}$. The outer target is initially attached $\left(T_{e} \sim 25 \mathrm{eV}\right)$. Although no neutral flux measurements at the inner divertor were available for this shot (gauges not yet installed), it can be expected a flux distribution similar to that in figure 3 at the end of the density ramp. Again $\Gamma_{D, F 1}$ increases fast and abruptly compared to the time scale of the $\bar{n}_{e}$ rise indicating a change of divertor plasma regime. The increase in the subdivertor flux is correlated with an even stronger rise of the flux in front of the outer target by a factor of ten reaching the subdivertor value of $\approx 9 \times 10^{22}$ Deuterium atoms $m^{-2} s^{-1}$. No significant delay of $\Gamma_{D, F 1}$ with respect to $\Gamma_{D, F 8}$ could be deduced. In figure 5 we plot the ratio $\Gamma_{D, F 1} / \Gamma_{D, F 8}$ for the $\mathrm{W}$ set of discharges as in figure 2. At the lower density, in the low recycling regime, the neutral flux below the dome is $3-5$ times larger than the flux close to the outer strike point. When the outer divertor approaches detachment (see figure 2) the flux ratio becomes close to 1 . The interpretation is similar to the previous example at low $\bar{n}_{e}$ : the low $T_{e}(<10 \mathrm{eV})$ at the outer divertor leg allow $\Gamma_{D, F 1}$ and $\Gamma_{D, F 8}$ to equalise. With the same argument, when both target plasmas are detached at $\bar{n}_{e}>5.5 \times 10^{19} \mathrm{~m}^{-3}$, we may expect the neutral fluxes 
in the lower divertor to be equal $\left(\Gamma_{D, F 1} \approx \Gamma_{D, F 19} \approx \Gamma_{D, F 8}\right)$ and the poloidal distribution to be uniform. This will be verified with future experiments. Also, since the region around target plates are the major neutrals sources, the simultaneous increase of $\Gamma_{D, F 1}$ and $\Gamma_{D, F 8}$ suggests that the neutral gas in the divertor is dominated by the outer target when the outer divertor plasma is detached.

\section{Comparison of ion and neutral particle fluxes at the outer target}

For data consistency check and comparison with numerical codes it is important to measure detailed profiles of the ion and neutral particle fluxes on the target around the strike point with independent methods. In AUG the neutral fluxes at the target are obtained by combining spectroscopic measurements of $D_{\alpha}$ radiation with atomic data under the assumption of dominant recycling and by pressure gauge \#8 (see figure 1). The atomic data is evaluated using $n_{e}$ and $T_{e}$ from Langmuir probes on the target. The profile from a single gauge is obtained by sweeping the outer strike point along the target during the discharge. The time is then re-mapped into the distance between the gauge position and the strike point. Since for low densities attached divertor plasmas conditions the recycled particles are proportional to the ion flux hitting the target surface, a strong link between $\Gamma_{+}$and $\Gamma_{D}$ profiles at the target is expected. Figure $6 \mathrm{a}$ ) and $6 \mathrm{~b}$ ) show the target fluxes for a low $\left(\bar{n}_{e}=2.8 \times 10^{19} \mathrm{~m}^{-3}\right)$ and medium $\left(\bar{n}_{e}=3.9 \times 10^{19} \mathrm{~m}^{-3}\right)$ plasma density with attached outer divertor. $\Gamma_{+}$and $\Gamma_{D}$ profiles are peaked in the SOL region close to the separatrix and are higher for higher density. The neutral fluxes from the gauge and $D_{\alpha}$ are in reasonable agreement in terms of absolute value but 
the profile from the ionisation gauge is generally flatter. An explanation could be the poor resolution of the gauge, about $1-1.5 \mathrm{~cm}$, which flattens the profile due to the finite size of the chimney $(0.7 \mathrm{~cm}$ diameter $)$, its position relative to the tiles surface and the shape of the tiles. However this effect appears to be small as shown by the convoluted $D_{\alpha}$ in figure 6a (green stars). Special tiles are in preparation to further investigate this effect. The ion flux is larger than the neutral flux by a factor of 2-3. At present the reasons for this difference are not understood and will have to be investigated more in details. In plasmas with higher density and high recycling a large volumetric (reionisation and recombination) reaction level is expected. The evaluation of $\Gamma_{D}$ from $D_{\alpha}$ under these conditions requires the knowledge of $n_{e}$ and $T_{e}$ along the lines of sight which is in general not available on AUG. Thus no agreement between different evaluations of neutral fluxes is expected in this case. Indeed we find that the $D_{\alpha}$ is about twice the neutral flux from the gauge (see figure 6c). The ion and $D_{\alpha}$ flux profiles are still peaked, with a maximum well inside the scrape-of-layer. 1-2 cm away from this peak $\Gamma_{D}$ is much larger than $\Gamma_{+}$ indicating that the recycling does no longer occur exclusively on the target surface.

\section{Conclusions}

Recycling characteristics similar for W-coated and CFC target plates for the same averaged plasma density. The density threshold for outer target detachment stay the same (within 10\%) and, at constant $\bar{n}_{e}$, neutral fluxes change maximum by a factor of 2 . The poloidal distribution of neutral particle fluxes in the divertor changes strongly with the plasma density. At low and medium 
densities it is highly asymmetric being larger at the inner target, especially after detachment of the inner target. At higher plasma density, when also the plasma at the outer target detaches, the neutral fluxes are larger in the outer divertor and appears to dominate the subdivertor flux. As a consequence the full neutrals distribution should be used to constrain the simulations or when comparing measurements and simulations. In attached plasma condition, the profiles of neutral particle flux around the strike point from $D_{\alpha}$ measurements and ion gauge agree within uncertainties as expected. Ion fluxes are typically 2-3 larger than neutral fluxes. This difference is at present unexplained and it has to be investigated in the future.

\section{References}

[1] M. Wischmeier et al., Contrib. Plasma Phys., 48, No. 1-3, 249-254 (2008)

[2] A.V. Chankin et al., Plasma Phys. Control. Fusion, 48 (2006) 839-868.

[3] Haas G., Bosch H.-S., Vacuum 51 (1998), p. 39-46.

[4] Neu R. et al., Plasma Phys. and Control. Fusion, 49(2007) B59-B70

[5] G.M. McCracken et al., Nuclear Fusion, 38(4), 1998

[6] A. Kallenbach et al., Nuclear Fusion, 43, 2003

[7] P.C. Stangeby, The Plasma Boudaries of Magnetic Fusion Devices, Plasma Physics Series, IOP, 2000. 


\section{$5 \quad$ Figure captions}

Fig. 1. ASDEX Upgrade poloidal cross-section and position of the fast pressure gauges. Newly installed gauges are \#8, \#19 and \#20. All gauges are installed in close fitting boxes with a small hole at one side through which neutrals enter the gauge. This side is marked by a tip on the symbols in the drawing of the poloidal distribution. Lines of sight of the $D_{\alpha}$ measurement at the outer target are shown with black lines.

Fig. 2. Above: Peak ion flux (blue dots) and electron temperature (green stars) at the outer target as function of plasma density in $1 M A$ ohmic discharges with $\mathrm{W}$. The red diamonds represent $\Gamma_{+}$in discharges with CFC targets. Below: subdivetor neutral flux density from gauge below the dome.

Fig. 3. Above: Plasma current (dashed line), line averaged plasma density (full line) and ECRH input power (dot-dashed line). Below: Time traces on neutral fluxes at the inner divertor $(F 19)$, inner column $(F 11)$, subdivertor $(F 1)$ and outer target $(F 8)$.

Fig. 4. Time evolution of the neutral fluxes during density ramp in the ohmic discharge \#22538. Subdivertor and outer target fluxes increases strongly and abruptly. The $\mathrm{X}$-point is formed at $\mathrm{t}=0.5 \mathrm{~s}$

Fig. 5. Ratio of the subdivertor to outer target fluxes as function of density in ohmic discharges with $1 M A$ of plasma current. 
Fig. 6. Comparison between the ion flux from Langmuir probes (dashed line) and the neutral particle fluxes from pressure gauge (full line) and $D_{\alpha}$ measurements (dash-dotted line). a) and b) are cases with low $\left(\bar{n}_{e}=2.8 \times 10^{19}\right)$ and medium $\left(\bar{n}_{e}=3.9 \times 10^{19}\right)$ density respectively and attached outer target. Green stars in fig. a) represent the convolute $D_{\alpha}$ profile with a box function of $1.5 \mathrm{~cm}$ width to simulate spatial resolution of the gauge. c) higher density $\left(\bar{n}_{e}=5.5 \times 10^{19}\right)$ with partially detached plasma and considerable recombination. The profiles are obtained through vertical sweep of the strike point at fixed plasma parameters in L-mode discharges.

\section{$6 \quad$ Figures}

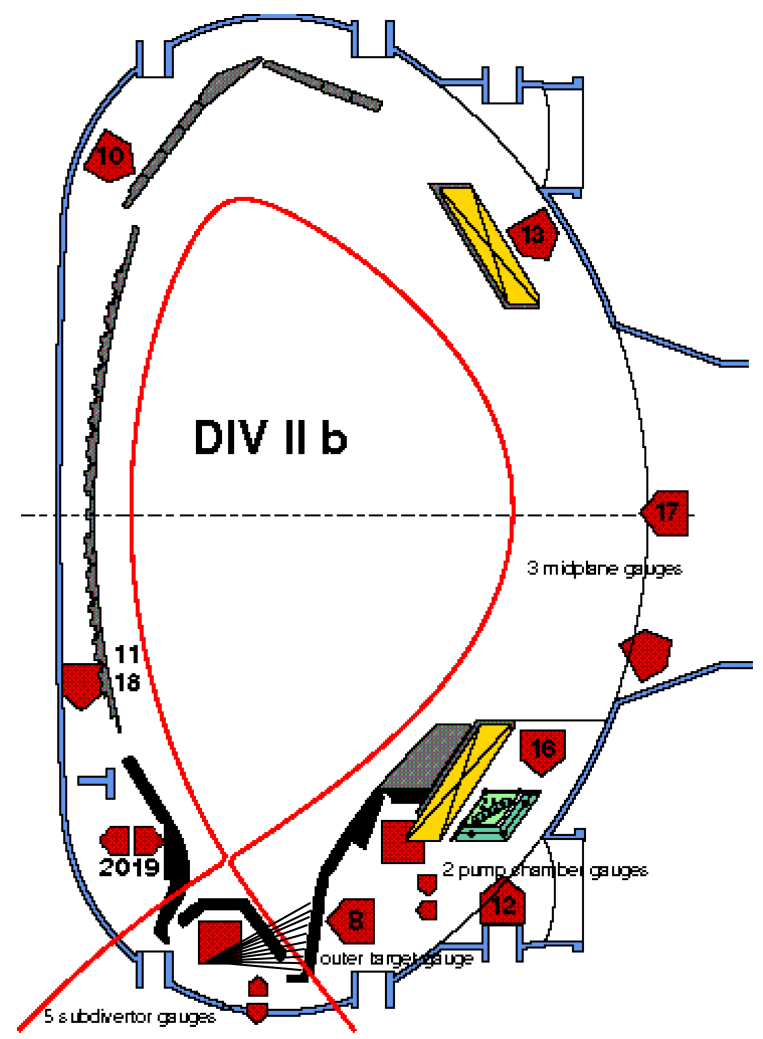

Figure 1 

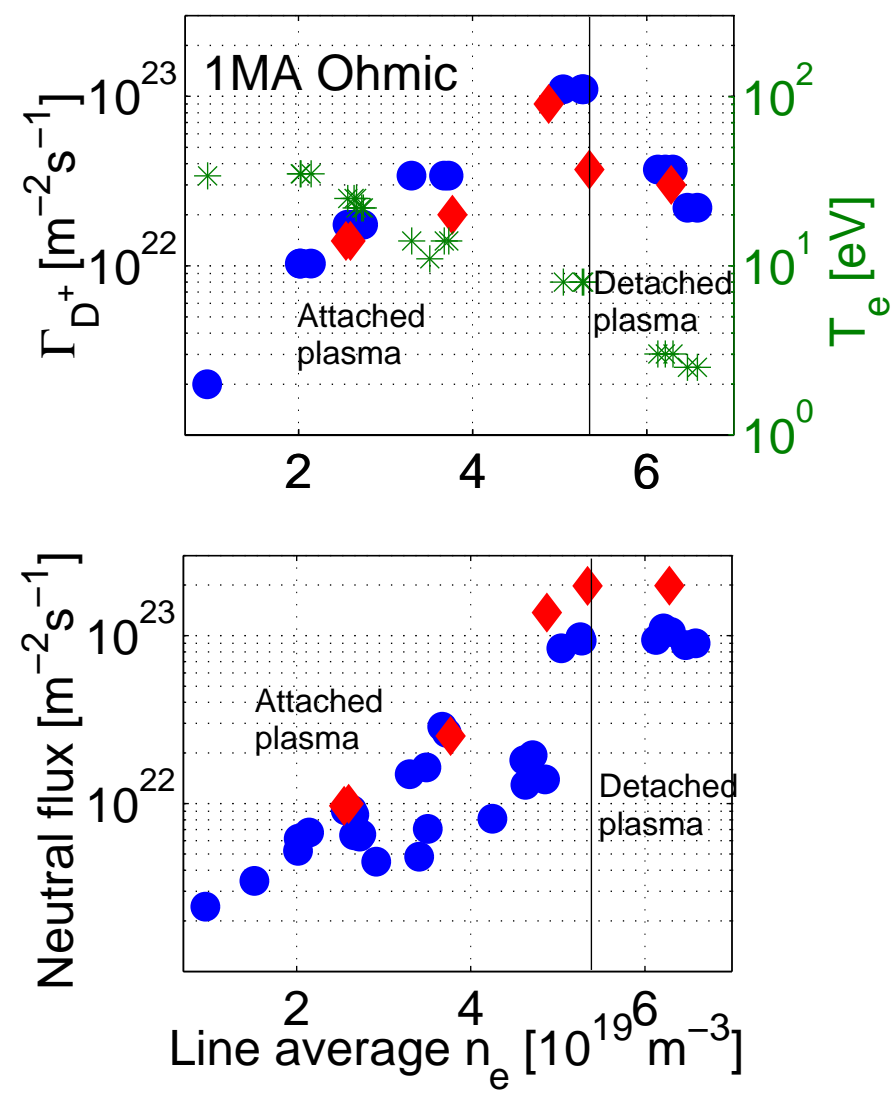

Figure 2

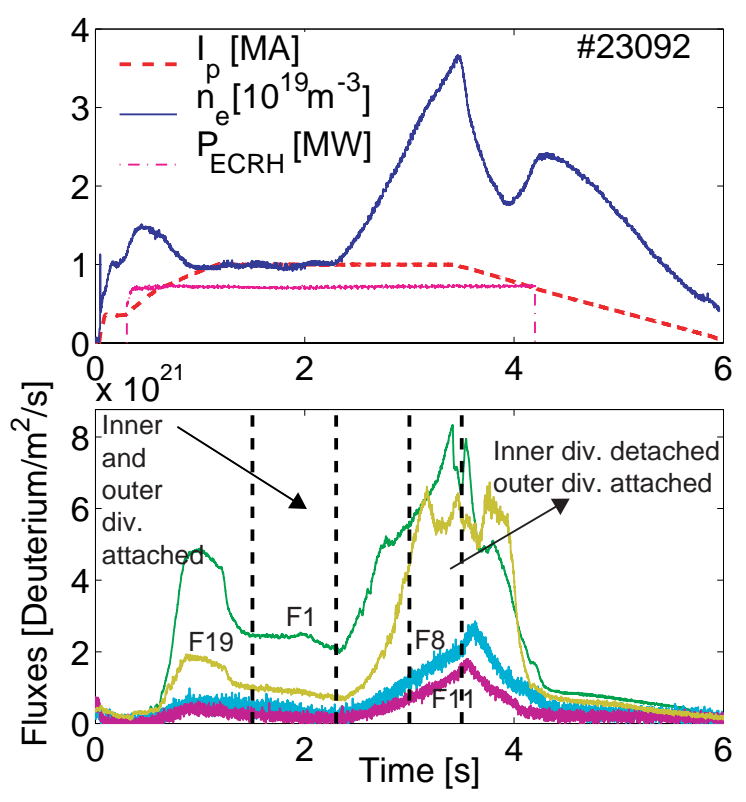

Figure 3 


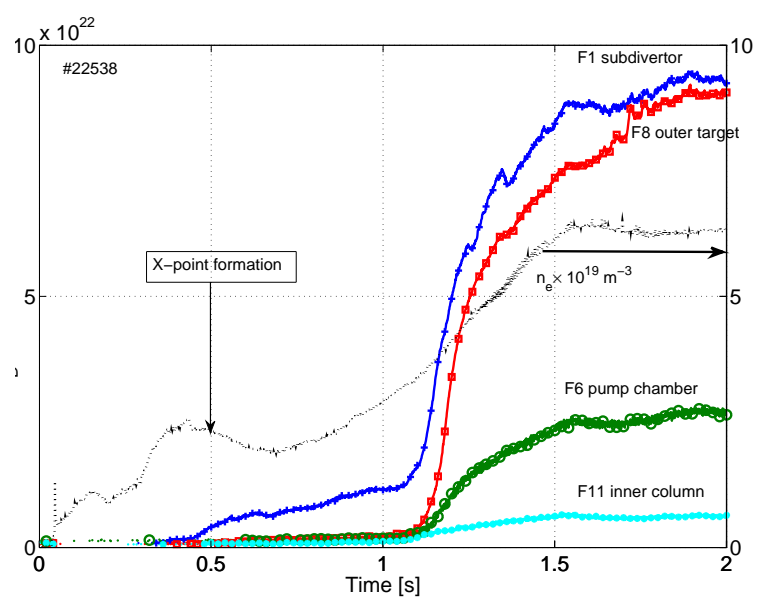

Figure 4

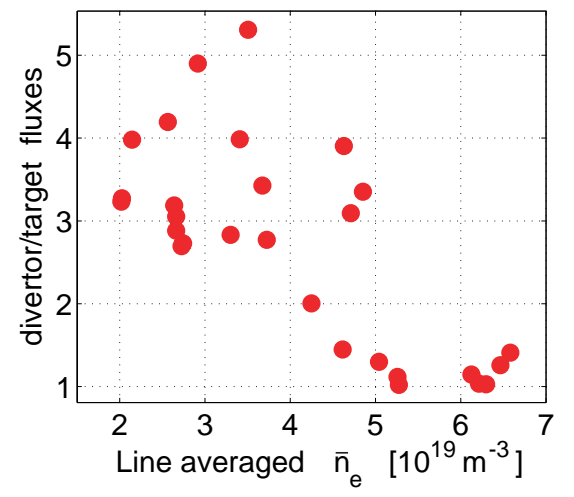

Figure 5 

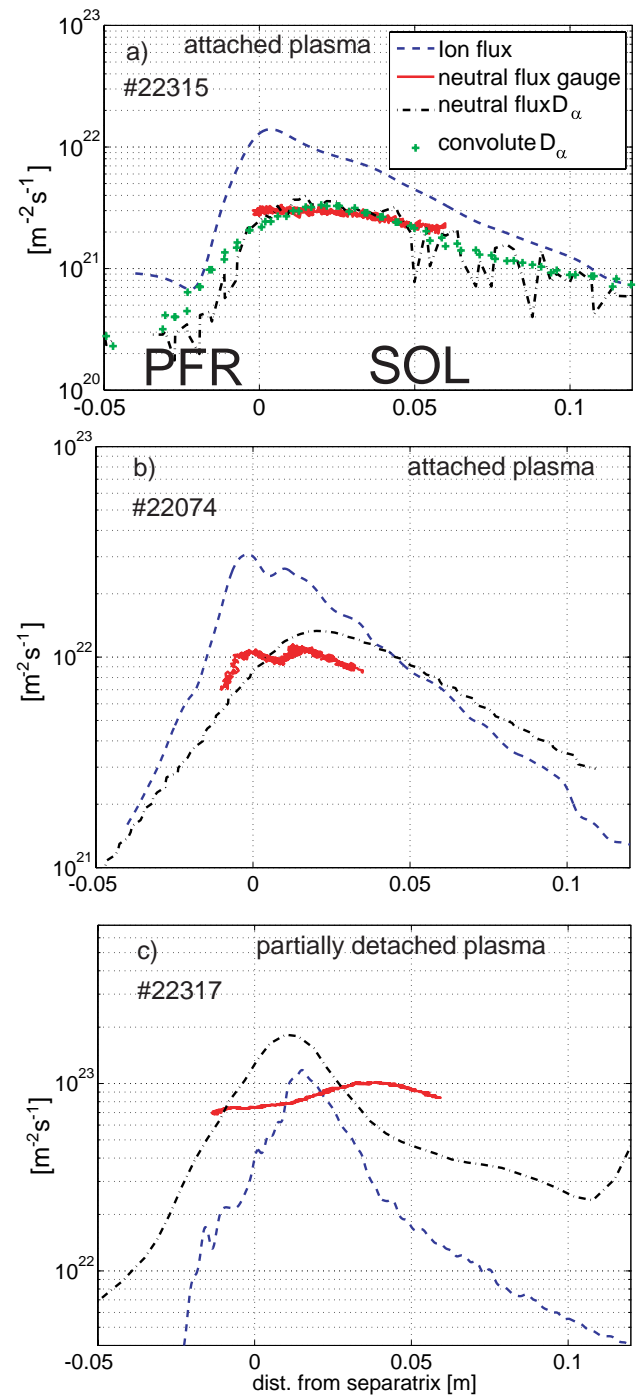

Figure 6 